\title{
Comparison of PD-L1, EGFR, ALK, and ROS1 Status Between Surgical Samples and Cytological Samples in Non-Small Cell Lung Carcinoma
}

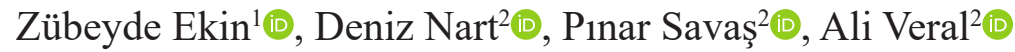 \\ ${ }^{1}$ Department of Pathology, Atatürk Training and Research Hospital, İzmir Katip Çelebi University, İzmir, Turkey \\ ${ }^{2}$ Department of Pathology, Ege University School of Medicine, İzmir, Turkey
}

Background: The expression levels of Programmed death ligand-1 (PD-L1), epidermal growth factor receptor (EGFR), anaplastic lymphoma tyrosine kinase gene (ALK), and proto-oncogene tyrosineprotein kinase 1 ROS (ROS1) are important for targeted treatment selection in advanced lung cancer. Most patients with lung cancer are diagnosed at an advanced stage and have no chance of surgery. For this reason, the accuracy and reliability of cytology samples for detecting those markers is important in patients whose histological sampling cannot be performed.

Aims: To test the compatibility of histological and cytological sample analysis results of EGFR, ALK, ROS1 and PDL-1 in patients with NSCLC and to determine the adequacy of cytological analysis for PD-L1 expression.

Study Design: Retrospective cross-sectional study.

Methods: The results of 231 patients whose PD-L1 was studied in 2018 were analyzed retrospectively. We excluded 11 inappropriate samples. A total of 220 samples were distributed as follows; 66 (30.0\%) cytology specimens, 64 (29.1\%) small histology biopsies, and $90(40.9 \%)$ surgical biopsies. EGFR, ALK, ROS1 and PD-L1 analysis were performed in $139,134,116$, and 220 patients, respectively. Samples containing $>400$ cells were considered suitable for molecular cytological study.

Results: A total of 154 (70.0\%) histological (surgical biopsy) and 66 $(30.0 \%)$ cytology samples were analyzed. There was no statistically significant difference between histological and cytological samples in terms of cellular adequacy for all molecular markers [EGFR: 93.7\% and $90.9 \%(P=.556)$, ALK: $97.8 \%$ and $95.3 \%(P=.436)$, ROS1: $89.9 \%$ vs. $91.9 \%(P=.729)$, PD-L1: $95.5 \%$ vs. $92.4 \%(P=.364)]$. There was no statistically significant difference in the expression positivity rates of all biomarkers between histological and cytological samples [EGFR: $9.0 \%$ vs. $2.5 \%(P=.018)$, ALK: $7.9 \%$ vs. $9.8 \%(P=.719)$, ROS1 : $1.4 \%$ vs. $2.9 \%(P=.591)$, PD-L1: $54.4 \%$ vs. $41.0 \%(P=.078)]$. Conclusion: The cellular adequacy of cytology specimens for molecular testing in patients with NSCLC is satisfactory. This study shows that EGFR, ALK, ROS-1 and PDL-1 expression rates in cytological samples are not statistically different from histological samples.

\section{INTRODUCTION}

Lung cancer (LC) is the most common global cause of cancer deaths in the 21 st century. Despite the development of cancer therapies, the treatment of LC is still challenging. As a class, non-small cell lung carcinoma (NSCLC) is the most prevalent subtype and accounts for nearly $75 \%$ of LCs.

Although LC screening programs have detected more patients with NSCLC at an early stage, a significant number of patients with NSCLC have metastatic disease or are locally advanced at diagnosis. ${ }^{2}$ The management and treatment of patients with advanced-stage NSCLC have shown great improvements. The development of effective systemic treatments for somatic mutations and rearrangements has revolutionized LC treatment. ${ }^{3}$ The primary treatment of metastatic NSCLC depends on the detection of some molecular driver mutations, such as those alterations in epidermal growth factor receptor (EGFR) and rearrangement of anaplastic lymphoma tyrosine kinase gene (ALK) and proto-oncogene tyrosine-protein kinase 1 ROS (ROS1). ${ }^{4}$ Currently, LC has become a prototype for solid tumors in regard to the success of targeted therapies. Testing for the EGFR mutation and the ALK and ROS1 mutations is required in the pretreatment algorithm in

Corresponding author: Zübeyde Ekin, Department of Pathology, Atatürk Training and Research Hospital, İzmir Katip Çelebi University, İzmir, Turkey

e-mail: zubeydeyildirimekin@gmail.com

Received: August 12, 2020 Accepted: June 17, 2021 •DOI: 10.5152/balkanmedj.2021.20086

Available at www.balkanmedicaljournal.org

ORCID iDs of the authors: Z.E. 0000-0002-9429-9394; D.N. 0000-0002-8100-6978; P.S. 0000-0002-3727-0480; A.V. 0000-0003-0343-9978.

Cite this article as:

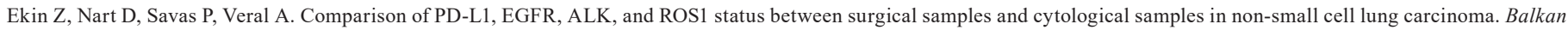
Med J. 2021;38(5):287-295.

Copyright@Author(s) - Available online at http://balkanmedicaljournal.org/ 
cases of advanced-stage LC. Compared with conventional platinum-based chemotherapy, patients using tyrosine kinase inhibitors have been reported to have longer survival, less toxicity, and a better quality of life. ${ }^{5}$

Advanced-stage patients with NSCLC without targetable genetic alterations have been limited to conventional therapies. Blockade of programmed cell death protein-1 (PD-1) and its ligand (programmed cell death ligand-1 [PD-L1]) using monoclonal antibodies such as pembrolizumab has been demonstrated to produce permanent responses in metastatic and locally advanced NSCLC, including squamous cell carcinoma. ${ }^{2}$ However, immune checkpoint inhibitors may trigger immune-related adverse effects, such as autoimmune disorders and pneumonitis. Therefore, biomarkers capable of identifying patients who are most likely to respond to immune checkpoint inhibitors are critical for the therapy. Tumors with high PD-L1 expression show a better response to anti-PD-1 therapy. ${ }^{6}$ More recently, the use of pembrolizumab was approved for first-line therapy in advanced NSCLC, immunohistochemically showing a PD-L1(clone $22 \mathrm{C} 3$ ) tumor ratio score (TPS) of $1.0 \%$ or more. ${ }^{7}$

The pathologic diagnosis is mostly made with small biopsies or cytologic material in patients with advanced-stage LC. ${ }^{8}$ Despite the challenge constantly posed by the presence of small and limited histologic samples, genotyping of tumor samples is broadly accepted on cytologic and small histologic samples. ${ }^{9}$ According to the guidelines, EGFR and ALK testing on cytologic specimens and cell blocks is advocated. ${ }^{10,11}$

In the current study, the use of PD-L1 quantification was investigated in cytologic samples of NSCLC.

\section{MATERIAL AND METHODS}

Patients and Specimens

We performed a retrospective analysis of molecular pathology reports of patients with PD-L1-stained NSCLC at our center from January 2018 to December 2018. To obtain more accurate results, samples that had not undergone mutation analysis were excluded. Formalin-fixed paraffin-embedded (FFPE) tissues, cell blocks (CB), and cytologic slides (Figure 1) were used for these molecular mutation tests. The study was approved by the Ethics Committee of Ege University (Approval No.: 21-2T/55).

Cytologic slides were obtained using image-guided fine-needle aspiration (FNA), FNA only, transthoracic aspiration, thoracentesis, and pericardiocentesis. Expelled tissues from these aspirates were processed as smears, and residual samples were prepared for liquid-based cytology using a ThinPrep5000 automated slide processor (Hologic, Marlborough, MA, USA), as described in the kit's instructions. All slides were fixed in $90 \%$ ethanol and stained using the Papanicolaou method. Thoracentesis and pericardiocentesis specimens were prepared for liquid-based cytology.

CBs were prepared as follows: Cytologic specimens were spun down and the pellets were embedded into paraffin blocks. If the cytologic specimen was in ThinPrep solution, the CB was prepared from residual ThinPrep test samples using the plasma thrombin

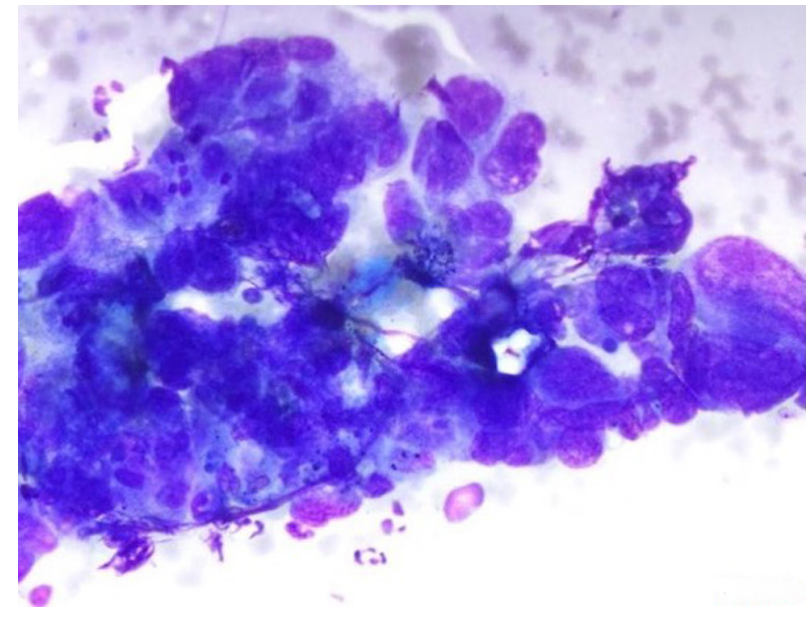

FIG. 1. Cytologic findings. Non-small cell lung carcinoma. Clusters of epithelial cells with high nuclear/cytoplasmic ratio are observed $(\times 400)$.

method. ${ }^{12}$ The histologic samples were embedded into paraffin after fixation in $10 \%$ neutral buffered formalin. The overall fixation time was determined as 4 hours and 48 hours depending on the specimens (Figure 2). Samples were prepared and stained with hematoxylin-eosin (H\&E) and assessed under a microscope. Samples containing $>400$ cells were considered suitable for molecular study.

We evaluated pathology reports from 231 patients with NSCLC analyzed for EGFR, ALK, ROS1, and PD-L1 expression in our center from January 2018 through December 2018. However, we did not perform mutation analysis in 11 cases because of insufficient and poor quality DNA. A total of 220 cases were included. One hundred thirty (59.1\%) patients who were tested for EGFR, ALK, ROS1, and PD-L1 expression were diagnosed in our department and 90 $(40.9 \%)$ patients were diagnosed in other laboratories and referred for mutation analysis to our laboratory. One hundred seventynine samples $(81.4 \%, 179 / 220)$ were taken from the lung and 41 $(18.6 \%, 41 / 220)$ were from other locations. There were $66(30.0 \%$,

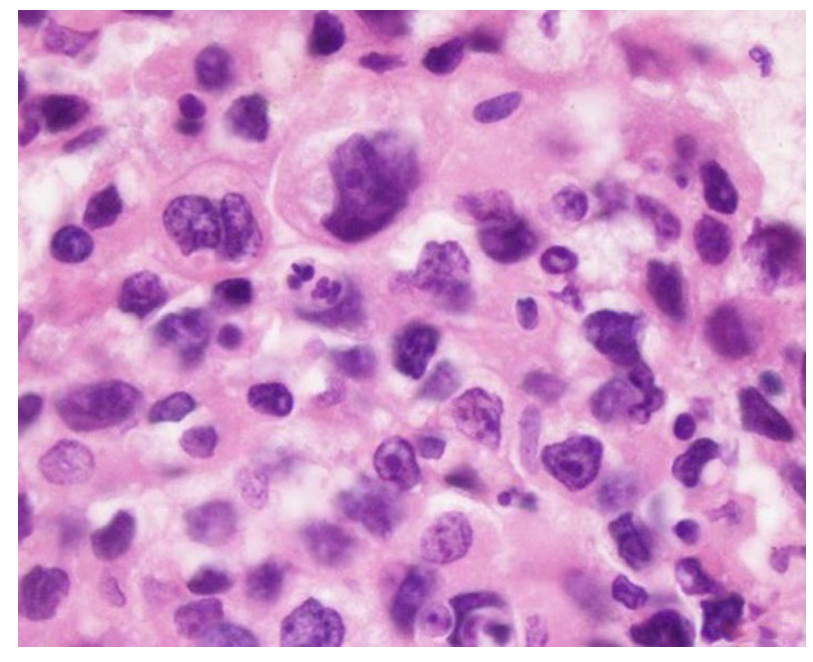

FIG. 2. Cell block findings of non-small cell carcinoma $(\times 400)$. 
66/220) cytology specimens, 64 (29.1\%, 64/220) small histologic biopsies, and 90 (40.9\%, 90/220) surgical biopsies. Small histologic biopsy specimens were included in 19 (19/64, 29.6\%) CT-guided needle biopsy samples, 35 (35/64, 54.6\%) endobronchial forceps biopsy specimens, and $10(10 / 64,15.8 \%)$ other histologic biopsy samples. Cytology samples were obtained from 27 (27/66, 40.9\%) EBUS-FNA specimens, 3 (3/66, 4.5\%) other FNA specimens, 29 $(29 / 66,43.9 \%)$ transthoracic aspiration specimens, and 7 (7/66, $10.7 \%$ ) thoracentesis or pericardiocentesis specimens. Specimens were diagnosed as adenocarcinoma ( $n=151,68.6 \%)$, NSCLC not otherwise specified (NSCLC-NOS) ( $n=26,11.8 \%$ ), squamous cell carcinoma $(n=41,18.6 \%)$, and other carcinomas $(n=2,1.0 \%)$. The detailed clinicopathologic specimens and patient information are defined in Table 1. EGFR, ALK, ROS1 and PD-L1 analysis were performed in 139, 134, 116, and 220 patients, respectively.

\section{Epidermal Growth Factor Receptor Analysis}

Formalin-fixed paraffin-embedded tissues, CBs, and stained cytologic slides were used for EGFR mutation analysis. The pathologist chose the tumor or tumor-rich fields for EGFR mutation analysis.

TABLE 1. Clinical and Pathologic Details of the Patients and Specimens

\begin{tabular}{|c|c|}
\hline Characteristics & \\
\hline Specimens & $220(100 \%)$ \\
\hline \multicolumn{2}{|l|}{ Site } \\
\hline Lung & $179(81.4 \%)$ \\
\hline Regional lymph nodes & $24(10.9 \%)$ \\
\hline Pleura/pericardium/mediastinum & $12(5.5 \%)$ \\
\hline Distant metastasis & $5(2.3 \%)$ \\
\hline Brain & $2(0.9 \%)$ \\
\hline Skin & $1(0.4 \%)$ \\
\hline Bone & $1(0.4 \%)$ \\
\hline Adrenal gland & $1(0.4 \%)$ \\
\hline \multicolumn{2}{|l|}{ Type } \\
\hline Surgical resection & $90(40.9 \%)$ \\
\hline Small histologic biopsy & $64(29.1 \%)$ \\
\hline Cytologic biopsy & $66(30.0 \%)$ \\
\hline \multicolumn{2}{|l|}{ Method } \\
\hline Histology & $154(70.0 \%)$ \\
\hline Cytology & $66(30.0 \%)$ \\
\hline Patients & $220(100 \%)$ \\
\hline \multicolumn{2}{|l|}{ Sex } \\
\hline Male & $167(75.9 \%)$ \\
\hline Female & $53(24.1 \%)$ \\
\hline \multicolumn{2}{|l|}{ Diagnosis } \\
\hline Adenocarcinoma & $151(68.6 \%)$ \\
\hline Squamous cell carcinoma & $41(18.6 \%)$ \\
\hline NSCLC, NOS & $26(11.8 \%)$ \\
\hline Other carcinomas & $2(1.0 \%)$ \\
\hline
\end{tabular}

NSCLC-NOS, non-small lung cancer-not otherwise specified.

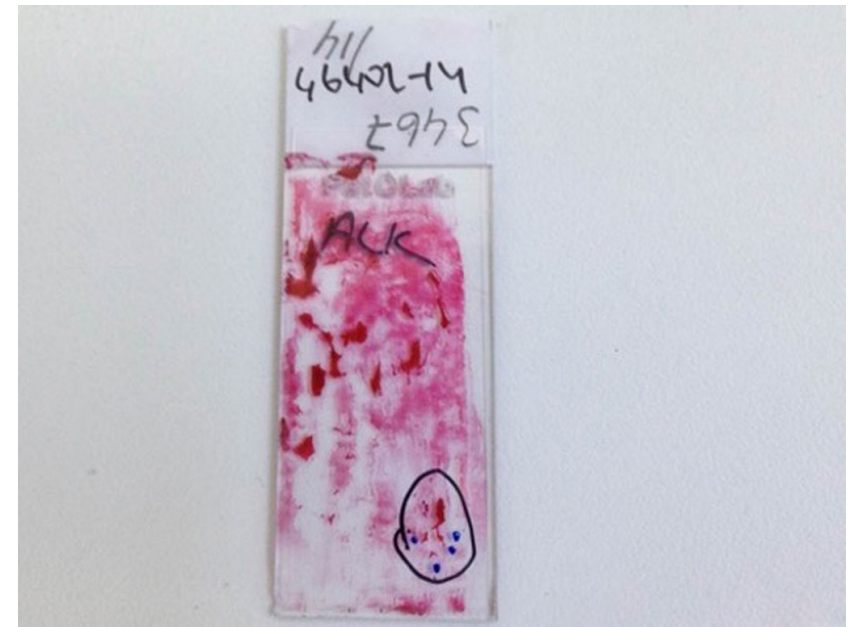

FIG. 3. Smear slide is marked on hematoxylin and eosin-stained sections to select areas rich in tumor or consisting of tumor.

These fields were macrodissected and eliminated as many stromal or non-tumoral fields (Figure 3). Sterilized scalpels were used to avoid contamination.

DNA was obtained from CBs and FFPE using a QIAamp DNA FFPE kit (Qiagen, Germany) based on the kit instructions. For H\&E, Giemsa or Papanicolaou-stained cytologic slides, genomic DNA was isolated using the phenol-chloroform method. Specimens with $>25 \%$ tumor cell content were used for the test.

Mutations of exons 18, 19, 20, and 21 were analyzed using the therascreen EGFR Pyro Kit on an Applied Rotor-Gene Q 2plex realtime polymerase chain reaction (PCR). All samples were assessed for mutation analysis according to the manufacturer's instructions.

\section{ALK and ROS1 Mutation Analysis}

FFPE tissues, CBs, and stained cytology slides were used for ALK and ROS1 mutation testing. On stained cytology slides and H\&Estained sections, the pathologist marked the tumor or the tumorrich areas. Technicians applied ALK and ROS1 by considering these identified regions. ALK rearrangement was evaluated using a Vysis ALK Break Apart fluorescence in situ hybridization (FISH) kit (Abbott Molecular, IL, USA) and the Vysis ROS1 FISH Break Apart Kit (Abbott Molecular, IL, USA), as described in the instructions. FFPE specimens were manually processed using the Post Hybridization Wash Buffer Kit in accordance with the manufacturer's instructions. FISH images were uploaded on the BioView AllegroPlus system and remotely assessed via BioView SoloWeb.

ALK rearrangement was evaluated based on the package insert for the Vysis ALK Break Apart FISH Kit. At least 50 tumor cells were counted. Cells showing break-apart signals (a minimum of 2 signal diameters apart) or a single orange signal for ALK and a single green signals for ROS1 were considered positive (Figure 4). A sample with more than $50 \%$ positive nuclei was considered as positive for ROS1 or ALK rearrangement, and a sample with less than $10 \%$ positive nuclei was considered as negative for ROS1 or ALK rearrangement. Any sample with 10\% 


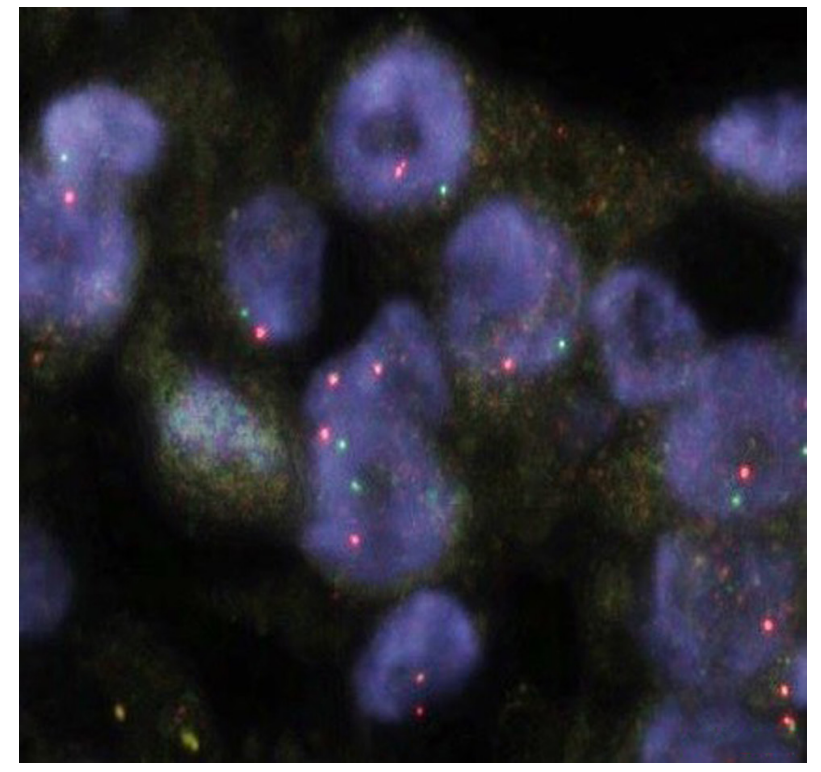

FIG. 4. ALK FISH of a cell block of good quality. Break-apart signals are observed $(\times 400)$.

to $50 \%$ positive nuclei was classified as equivocal. If the sample was equivocal, the slide was evaluated by a second reader who selected an extra 50 cells to be counted. For these samples, tumor cells with more than $15 \%$ positive nuclei were defined as positive (Figure 5), and those with less than $15 \%$ positive nuclei were considered negative.

\section{Immunohistochemical PD-L1 Expression Analysis and Scoring for PD-L1}

FFPE tissues and CBs were used for PD-L1 testing. Tissue sections of $4 \mu \mathrm{m}$ were cut and mounted on positively charged slides. PD-L1 staining was performed based on the datasheet instructions (Dako PD-L1 22C3 PhamDx, Dako North America Inc., Carpinteria, CA).

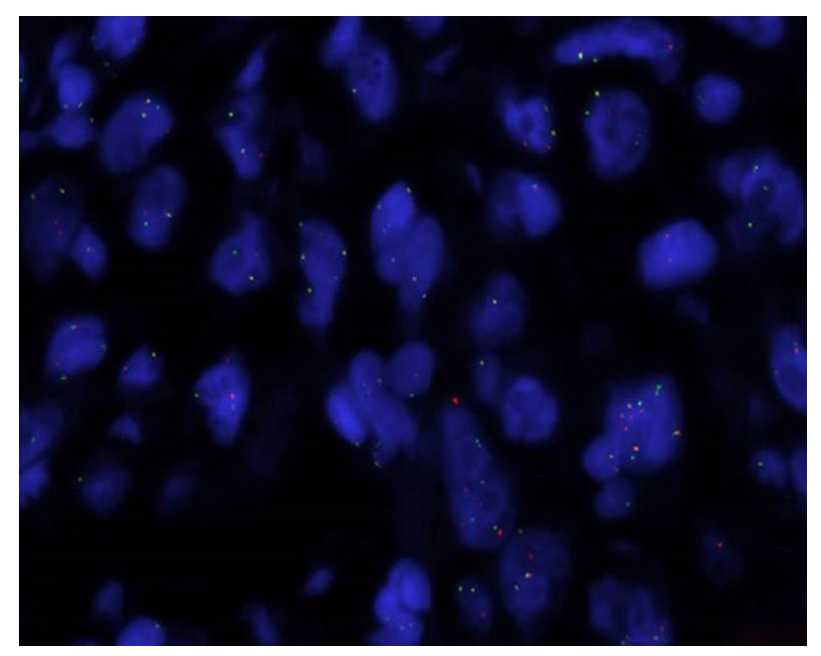

FIG. 5. ROS1 FISH of a conventional cytologic smear with good fluorescent signals $(\times 400)$.

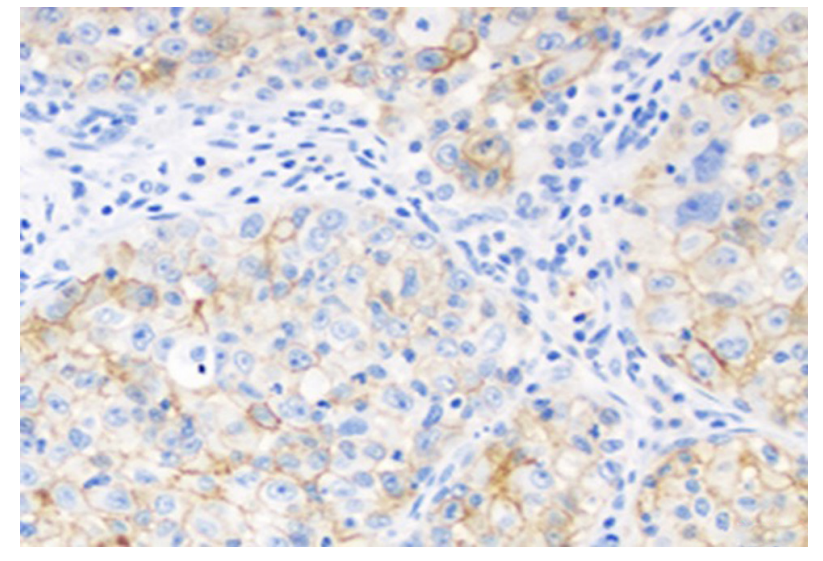

FIG. 6. Squamous cell carcinoma with high expression of PD-L1 $(\times 400)$

PD-L1 immunostained slides were re-evaluated by 2 experienced pathologists, independently. Controversial cases were re-analyzed and resolved through consensus. At least 100 viable and wellpreserved cancer cells were accepted as adequate. PD-L1 expression was assessed independently both in tumor cells (TCs) and tumor-infiltrating immune cells (ICs) in both cytologic and surgical samples. Membranous-stained TCs and ICs were defined as positive. PD-L1 expressions of tumor cells were evaluated as in the report of Reck et al. ${ }^{13}$ According to the recommendations, cancer cells with weak $(1+)$, moderate $(2+)$ or strong $(3+)$ membranous staining were considered as positive. The Tumor Proportion Score (TPS) was categorized as $<1 \%$ (no PD-L1 expression), 1-49\% (PD-L1 expression), and $50 \%$ (high PD-L1 expression) cancer cells. At least 100 viable cancer cells were evaluated as adequate (Figure 6). ICs were assessed as $<10 \%$, no PD-L1 expression; and 10\%, PD-L1 expression.

\section{Statistical Analysis}

The numerical and categorical variables are summarized as mean \pm standard deviation, frequency, and percentage, respectively. We compared parametric variables using $t$-tests and categorical variables using the chi-square test. The correlation between PD-L1 and other variables was assessed with the Pearson correlation test. A value of $P<.05$ was considered statistically significant. When we performed post-hoc power analysis (based on a rate of PD-L1 accuracy of $99 \%$ for histology and $95 \%$ for cytology and alpha of 0.05 with a sample size of 220), the power of the study was calculated as $96.6 \%$. Statistical analyses were performed using the SPSS version 19.0 for Windows software package (IBM SPSS Corp.; Armonk, NY, USA).

\section{RESULTS}

The median age of the patients was 64 (range, 28-91) years.

EGFR analysis was performed in 139 patients. A total of 40 of 44 (90.9\%) cytology samples were adequately cellular for the EGFR analysis, including 21 of $24(87.5 \%)$ smears and 19 of $20(95.0 \%)$ CBs. In the study, 35 of $40(87.5 \%)$ small histologic biopsy samples and 54 of $55(98.2 \%)$ surgical resection specimens were adequately cellular for EGFR analysis $(P=.116)$. ALK analysis was performed in 134 cases. A total of 41 of $43(95.3 \%)$ cytology samples were 
TABLE 2. Adequacy Rate According to Specimen Type

\begin{tabular}{lcccc}
\hline $\begin{array}{l}\text { Specimen } \\
\text { Type }\end{array}$ & $\begin{array}{c}\text { No. EGFR } \\
\text { Adequate }(\%)\end{array}$ & $\begin{array}{c}\text { No. ALK } \\
\text { Adequate } \%)\end{array}$ & $\begin{array}{c}\text { No. ROS1 } \\
\text { Adequate }(\%)\end{array}$ & $\begin{array}{c}\text { No. PD-L1 } \\
\text { Adequate } \\
(\%)\end{array}$ \\
\hline Cytology & $40 / 44(90.9 \%)$ & $41 / 43(95.3 \%)$ & $34 / 37(91.9 \%)$ & $\begin{array}{c}61 / 66 \\
(92.4 \%)\end{array}$ \\
Small biopsy & $35 / 40(87.5 \%)$ & $36 / 38(94.7 \%)$ & $27 / 33(81.8 \%)$ & $59 / 64$ \\
& & & & $(92.2 \%)$ \\
Resection & $54 / 55(98.2 \%)$ & $53 / 53(100 \%)$ & $44 / 46(95.7)$ & $88 / 90$ \\
& & & & $(97.8 \%)$ \\
Total & $129 / 139$ & $130 / 134$ & $105 / 116$ & $208 / 220$ \\
& $(92.8 \%)$ & $(97.0 \%)$ & $(90.5 \%)$ & $(94.5 \%)$ \\
\hline
\end{tabular}

EGFR, epidermal growth factor receptor; ALK, anaplastic lymphoma tyrosine kinase gene; ROS1, proto-oncogene tyrosine-protein kinase 1 ROS; PD-L1, programmed cell death protein ligand-1.

adequately cellular for ALK analysis, including 21 of 22 smears (95.4\%) and 20 of $21(95.2 \%)$ CBs. A total of 36 of $38(94.7 \%)$ small histologic biopsy samples and 53 of $53(100 \%)$ surgical resection samples were adequate for ALK analysis $(P=.256)$. ROS1 analysis was performed in 116 cases. A total of 34 of 37 (91.9\%) cytology samples were adequately cellular for ROS1 analysis, including 17 of $19(89.4 \%)$ smears and 17 of $18(94.4 \%)$ CBs. A total of 27 of 33 $(81.8 \%)$ small histologic biopsy samples and 44 of $46(95.7 \%)$ surgical resection samples were adequately cellular for ROS1 analysis $(P=.111)$. PD-L1 analysis was performed in 220 cases. Further, 61 of $66(92.4 \%)$ cytology specimens (all CBs), 59 of $64(92.2 \%)$ small histologic biopsy samples, and 88 of $90(97.8 \%)$ surgical resection samples were adequate for PD-L1 analysis $(P=.213)$. No significant difference was found between the specimen types. The adequacy rate according to specimen type is shown in Table 2.

There was also no difference between histologic and cytologic specimens; 40 of $44(90.9 \%)$ cytologic specimens and 89 of 95 $(93.7 \%)$ histologic specimens were adequately cellular for EGFR analysis $(P=.556)$. A total of of 43 cytology specimens $(95.3 \%)$ and 89 of $91(97.8 \%)$ histologic specimens were adequately cellular for ALK analysis $(P=.436)$; 34 of $37(91.9 \%)$ cytologic specimens and 71 of $79(89.9 \%)$ histologic specimens were adequately cellular for ROS1 analysis $(P=.729)$; 61 of $66(92.4 \%)$ cytologic specimens, and 147 of $154(95.5 \%)$ histologic samples were adequately cellular for PD-L1 analysis $(P=.364)$.

\section{EGFR, ALK, ROS and PD-L1 Positivity Rates According to the Cytologic and Histologic Specimen Subgroups}

EGFR mutation was negative in 120 samples (86.3\%), positive in $9(6.5 \%)$, and unsatisfactory for evaluation in 10 samples (7.2\%). EGFR positivity was $9.0 \%(n=8)$ in histologic specimens compared with $2.5 \%(n=1)$ in cytologic specimens $(P=.181)$. EGFR positivity was $0.0 \%(n=0)$ in CBs compared with $4.1 \%$ $(n=1)$ in smear specimens $(P=.076)$. ALK mutation was negative in 119 samples $(88.8 \%)$, positive in $11(8.3 \%)$, and unsatisfactory for evaluation in 4 samples $(2.9 \%)$. ALK positivity was $7.9 \%$ $(n=7)$ in histologic specimens compared with $9.8 \%(n=4)$ in cytologic specimens $(P=.719)$. ALK positivity was $9.5 \%(n=2)$ in CBs compared with 9.0\% $(n=2)$ in smear specimens $(P=.197)$. ROS1 mutation was negative in 103 samples $(88.7 \%)$, positive in
TABLE 3. EGFR, ALK, ROS, PD-L1 Positivity Rate According to Cytological and Histological Specimen Subgroups

\begin{tabular}{lccc}
\hline Molecular Test & No. Histology $(\%)$ & No. Cytology $(\%)$ & $P$ \\
\hline EGFR positivity & $8(9.0 \%)$ & $1(2.5 \%)$ & .181 \\
ALK positivity & $7(7.9 \%)$ & $4(9.8 \%)$ & .719 \\
ROS1 positivity & $1(1.4 \%)$ & $1(2.9 \%)$ & .591 \\
PD-L1 positivity & $80(54.4 \%)$ & $25(41.0 \%)$ & .078 \\
\hline
\end{tabular}

EGFR, epidermal growth factor receptor; ALK, anaplastic lymphoma tyrosine kinase gene; ROS1, proto-oncogene tyrosine-protein kinase 1 ROS; PD-L, programmed cell death protein ligand-1.

$2(1.9 \%)$, and unsatisfactory for evaluation in 11 samples $(9.4 \%)$. ROS1 positivity was $1.4 \%(n=1)$ in histologic specimens compared with $2.9 \%(n=1)$ in cytologic specimens $(P=.591)$. ROS1 positivity was $5.5 \%(n=1)$ in CBs compared with $0.0 \%(n=0)$ in smear specimens $(P=.068)$. There were no differences between all cytologic and histologic specimens.

Immunohistochemically, of PD-L1 expression (TPS $<1 \%$ ) was negative $(<1 \%$, no PD-L1 expression) in $103(46.8 \%)$ specimens, PD-L1 expression (TPS 1-49\%) in 56 (25.4\%), high PD-L1 expression (TPS $\geq 50 \%)$ in $49(22.2 \%)$, and unsatisfactory for evaluation in $12(5.5 \%)$ specimens. TPS $\geq 1 \%$ was $54.4 \%$ $(n=80)$ in histologic specimens compared with $41.0 \%(n=25)$ in cytologic specimens However, these relationships did not reach statistical significance $(P=.078)$. (Table 3$)$. There were also no statistical differences in the rate of PD-L1 positivity between other variables.

\section{EGFR, ALK, ROS and PD-L1 Positivity Rates According to Histology}

The positivity rate concordance of EGFR mutations, ALK-ROS1 rearrangement, and PD-L1 expression between tumor tissues and cytology samples was analyzed according to the histologic diagnoses. EGFR mutation was positive in $8.1 \%(n=8 / 99)$ of patients with adenocarcinoma, and $8.3 \%(n=1 / 12)$ of patients with NSCLC-NOS. Other histologic subtypes were negative for EGFR mutations $(P=.814)$. ALK rearrangement was positive in $6.7 \%$ $(n=7 / 104)$ of patients with adenocarcinoma, $27.3 \%(n=3 / 11)$ of patients with NSCLC-NOS, and 7.7\% $(n=1 / 13)$ of patients with squamous cell carcinoma. Other histologic subtypes were negative for ALK rearrangement $(P=.229)$. ROS1 rearrangement was positive in $2.2 \%(n=2 / 89)$ of patients with adenocarcinoma, and other histologic subtypes were negative for ROS1 rearrangement $(P=$ .947). PD-L1 expression was positive in $48.3 \%(n=70 / 145)$ of patients with adenocarcinoma, 40.9\% $(n=9 / 22)$ of patients with NSCLC-NOS, $64.1 \%(n=25 / 39)$ of patients with squamous cell carcinoma, and $100 \%(n=1 / 1)$ of patients with pleomorphic carcinoma. Other histologic subtypes were negative for PD-L1 expression $(P=.200)$. The EGFR, ALK, ROS1, and PD-L1 positivity rates according to histologic subgroups are shown in Table 4.

\section{PD-L1 Expression According to EGFR, ALK, and ROS1 Subgroups}

PD-L1 expression (TPS $\geq 1 \%$ ) was present in $44.4 \%$ of the samples $(n=4 / 9)$ with EGFR mutations, $63.6 \%$ of the samples $(n=$ 
TABLE 4. EGFR, ALK, ROS, and PD-L1 Positivity Rate According to Histological Subgroups

\begin{tabular}{|c|c|c|c|c|}
\hline & No. EGFR Positivity (\%) & No. ALK Positivity (\%) & No. ROS1 Positivity (\%) & No. PD-L1 Positivity (\%) \\
\hline Adenocarcinoma & $8(8.1 \%)$ & $7(6.7 \%)$ & $2(2.2 \%)$ & $70(48.3 \%)$ \\
\hline Squamous cell carcinoma & $0(0.0 \%)$ & $1(7.7 \%)$ & $0(0.0 \%)$ & $25(64.1 \%)$ \\
\hline NSCLC-NOS & $1(8.3 \%)$ & $3(27.3 \%)$ & $0(0.0 \%)$ & $9(40.9 \%)$ \\
\hline Pleomorphic carcinoma & $0(0.0 \%)$ & $0(0.0 \%)$ & $0(0.0 \%)$ & $1(100 \%)$ \\
\hline Other carcinoma & $0(0.0 \%)$ & $0(0.0 \%)$ & $0(0.0 \%)$ & $0(0.0 \%)$ \\
\hline
\end{tabular}

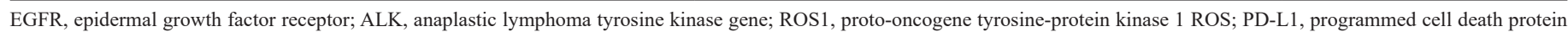
ligand-1; NSCLC-NOS, non-small lung cancer-not otherwise specified.

$7 / 11)$ with ALK rearrangement, and $50.0 \%$ of the samples $(n=1 / 2)$ with ROS1 rearrangement. There was no correlation between the PD-L1 expression scores and EGFR mutations, ALK, and ROS1 rearrangement (correlation coefficient, $P=.96, P=.33$, and $P=$ .96 , respectively).

PD-L1 Expression Rate of Tumor-Infiltrating Immune Cells PD-L1 expression of tumor-infiltrating ICs (TPS $>10 \%$ ) was present in $93.4 \%$ of histology samples $(n=57 / 147)$ and $6.6 \%$ of cytology samples $(n=4 / 61)$. There was a significant difference between cytologic specimens and histologic specimens $(P<.05)$. Tumor-infiltrating ICs' PD-L1 expression (TPS $>10 \%$ ) was present in $33.3 \%$ of samples $(n=3 / 9)$ with EGFR mutations, $18.2 \%$ of samples $(n=2 / 11)$ with ALK rearrangement, and $0 \%$ of samples $(n$ $=0)$ with ROS1 rearrangement. There was no correlation between tumor-infiltrating IC PD-L1 expression scores and EGFR mutations, and ALK and ROS1 rearrangement (Pearson's correlation coefficient, $P=.680, P=.385$, and $P=.376$ ). The pathologic characteristics of patients are shown as subdivided PD-L1 expressions in Table 5 .

\section{DISCUSSION}

Lung carcinoma can show various histologic findings. ${ }^{13}$ Generally, it is not easy to determine the histologic type of LC in small biopsy and cytology samples, and additional IHC staining is needed for the diagnosis. This often causes tissue loss for further assessment. In addition, due to recent advances in the molecular mechanism of LC and the success of targeted therapeutic agents, molecular tests are required to determine the optimal treatment procedure. ${ }^{14}$ Unfortunately, patients with NSCLC usually present with advancedstage disease. ${ }^{14}$ Cytology is a useful diagnostic technique that is preferred for obtaining biopsy samples in such patients. ${ }^{14}$ Moreover, patients with advanced-stage LC often have pleural and pericardial effusions, which have to be drained to reduce symptoms, and using effusion fluids and other cytologic materials will save unnecessary invasive procedures for IHC and molecular methods in patients lacking surgical material. ${ }^{12}$

Recently, anti-PD-1 agents have played a key role in advanced LC. The determination of PD-L1 expression is important in assessing the response to PD-1 and PD-L1. In this study, the compatibility of EGFR, ALK, ROS1, and PD-L between histologic and cytologic specimens were evaluated and it indicates that cytologic samples of NSCLC have sufficient cellularity in most cases to quantify PD-L1 expression.

Previous studies stated that cytologic specimens of NSCLC had adequate cellularity and were useful for molecular testing. ${ }^{15}$ In a study by Pang et al. ${ }^{16}$ including 670 patients with NSCLC, $11.9 \%$ of cytologic samples and $10.9 \%$ of histologic samples had insufficient tumor cells for EGFR testing. We have also found that cytologic

TABLE 5. Patient Pathologic Characteristics in Relation to PD-L1 Expression

\begin{tabular}{|c|c|c|c|c|c|c|}
\hline Characteristic & $\begin{array}{c}\text { Tumor Cell PD-L1 } \\
\text { Expression }>1 \%\end{array}$ & $\begin{array}{c}\text { Tumor Cell PD-L1 } \\
\text { Expression }<1 \%\end{array}$ & $P$ & $\begin{array}{l}\text { Tumor-Infiltrating } \\
\text { Immune Cell PD-L1 } \\
\text { Expression }>10 \%\end{array}$ & $\begin{array}{l}\text { Tumor-Infiltrating } \\
\text { Immune Cell PD-L1 } \\
\text { Expression }<10 \%\end{array}$ & $P$ \\
\hline \multicolumn{7}{|l|}{ Sex } \\
\hline Male & $49.7 \%(80)$ & $50.3 \%(81)$ & .673 & $27.3 \%(44)$ & $72.7 \%(117)$ & .241 \\
\hline Female & $53.2 \%(25)$ & $46.8 \%(22)$ & & $36.2 \%(17)$ & $63.8 \%(30)$ & \\
\hline \multicolumn{7}{|l|}{ Histology } \\
\hline Adenocarcinoma & $48.3 \%(70)$ & $51.7 \%(75)$ & .207 & $29.7 \%(43)$ & $70.3 \%(102)$ & .807 \\
\hline Squamous Cell Carcinoma & $64.1 \%(25)$ & $35.9 \%(14)$ & & $33.3 \%(13)$ & $66.7 \%(26)$ & \\
\hline NSCLC-NOS and Other & $41.6 \%(10)$ & $58.3 \%(14)$ & & $20.8 \%(5)$ & $79.2 \%(19)$ & \\
\hline \multicolumn{7}{|l|}{ Molecular Signature } \\
\hline EGFR mutation & $44.4 \%(4)$ & $55.6 \%(5)$ & .964 & $33.3 \%(3)$ & $66.7 \%(6)$ & .680 \\
\hline ALK rearrangement & $63.6 \%(7)$ & $36.3 \%(4)$ & .363 & $18.2 \%(2)$ & $81.8 \%(9)$ & .385 \\
\hline ROS1 rearrangement & $50 \%(1)$ & $50 \%(1)$ & .966 & $0 \%(0)$ & $100 \%(2)$ & .376 \\
\hline
\end{tabular}

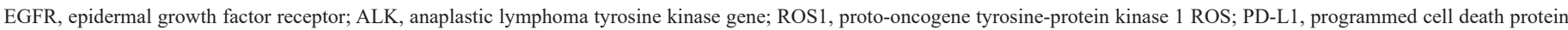
ligand-1; NSCLC-NOS, non-small lung cancer-not otherwise specified. 
specimens of NSCLC had adequate cellularity for FISH molecular testing (95.2\% for ALK, 97.4\% for ROS1). Many studies have used FISH for ALK on CBs yielding inconsistent data. Bravaccini et al. published $27(23 \%)$ cytologic materials, $3(2.5 \%)$ of which were cell block and $24(20.5 \%)$ of which were conventional smears, as insufficient for ALK FISH. ${ }^{17}$ On the other hand, other studies recorded an unsuccessfully approximate rate of $30 \%$ with CBs due to insufficient tumor cells in cytologic CB samples. ${ }^{18}$ Those different studies advised a standardization of CB preparation to optimize the use of cytologic specimens and to increase the value of the samples for molecular tests. It has been shown that the FISH performed on cytologic samples is more convenient and less problematic than that performed on histologic samples. ${ }^{19}$ To the best of our knowledge, there are insufficient data regarding FISH for ROS1 on cytologic samples. There is only one published study comprising 12 samples which has used FISH for ROS1 on cytologic smears. ${ }^{20}$ In a previous study, Noll et al. have reported that $92 \%$ of cytology CB samples had an adequate number of tumor cells. ${ }^{21}$ Sakakibara et al. ${ }^{22}$ have compared PD-L1 expression of EBUS-FNA and transbronchial biopsy samples of 97 patients with LC and they have determined that only one cytologic CB contained $<100$ tumor cells. Recently, Heymann et al. have shown great support for the use of cytology specimens for PD-L1 testing using the United States Food and Drug Administration (FDA)-approved pharmDx test. $^{23}$

Numerous studies have demonstrated that conventional cytologic smears (CCSs) were ideal for EGFR mutation analysis. ${ }^{24} \mathrm{CCSs}$ are compatible for PCR-based assays as they do not need formalin for fixation. An alcohol-based fixation step provides high-quality DNA for molecular testing. ${ }^{24}$ In addition, microdissection of the tumor area in the smear increases test success, particularly in samples with low cellularity. ${ }^{25,26}$ In our study, there was no EGFR positivity among $20 \mathrm{CBs}(0 \%)$ and there was only one (4.1\%) EGFR-positive case among 24 smear specimens, a finding which did not have any statistical difference $(P=.076)$. Some studies evaluated the use of CCSs for FISH for ALK testing. CCSs can be more effective than CBs for FISH for ALK testing. ${ }^{20,27}$ Some studies also have found that CCSs could be more effective for ROS1 testing compared with histologic samples. ${ }^{19,20}$ In the current study, no significant difference was found in ALK and ROS1 rearrangements between CBs and smear specimens $(P=.076, P=.197)$. CCSs are more suitable than $\mathrm{CBs}$, especially in samples with insufficient cellularity, because the use of CCSs exceeds some FISH-related limitations in FFPE samples; one of the major limitations of these samples is the loss of probe signals due to nuclear truncation. ${ }^{28}$ Five previous publications investigated the detection of ALK translocations in CB samples using FISH, 3 of which compared FISH with IHC. The authors have showed a significant correlation between FISH and IHC and they have concluded that the molecular tests applied to CBs had a high success rate and cytologic material can be an effective alternative to surgical biopsies in tests using IHC, FISH, or RT-PCR, and there were minor differences in specificities and sensitivities. ${ }^{29}$

In the current study, there was no difference in PD-L1 positivity when using different tissue samples including cytologic and histologic specimens (Table 3). This result is important because most patients with LC are diagnosed with cytology specimens. Due to tumor heterogeneity, there was concern about the comparison and reliability of PD-L1 test in cytology samples with histologic samples. ${ }^{30}$ There are conflicting results in publications where excisional histologic samples are compared with small samples. In a study involving 160 patients, PD-L1 expression was compared in paired biopsy and surgical excision specimens ${ }^{2}$ and significant discordance $(48.0 \%)$ was found between the 2 specimen types. In a recent study, no significant difference was found in PD-L1 positivity between small histologic biopsy samples (25.8\%) and surgical resection samples $(25.7 \%)$, and PD-L1 positivity was lower in histologic samples (25\%) compared with cytologic samples $(39 \%) .{ }^{23}$ In another study, PD-L1 expression results were compared in 86 paired cytologic $\mathrm{CBs}$ and histologic material using DAKO 28-8 and 22C3 clones, and a high degree of accordance was found between histologic and cytologic samples. ${ }^{31}$ These results suggest that the evaluation of PD-L1 expression in tumor cells could also be performed using cytologic CB samples, and PD-L1 expression could be a good alternative in the absence of surgical samples.

In our study, there was a significant difference in the expression of PD-L1 of ICs in various tissue samples, including cytology samples and histologic samples. There are several possible explanations for these findings. It is difficult to say that all ICs in cytologic material are tumor-related immune cells. In addition, there may be problems with the spatial heterogeneity of ICs and difficulty in distinguishing ICs from TCs. Rehman et al. ${ }^{32}$ found 94\% compatibility among pathologists in the evaluation of PD-L1 in TCs, but only $27 \%$ in IC PD-L1 expression. Pathologists showed excellent concordance for PD-L1 tumor scoring, but not for IC scoring. Similarly, Rimm et al. ${ }^{33}$ have reported that pathologists have shown poor concordance when scoring IC staining. A recent study found less interobserver agreement in terms of scoring expression of PD-L1 in ICs in cytology samples, as in previous reports. ${ }^{34}$ All these findings show that cytology samples are not appropriate for scoring expression of PD-L1 in ICs. Accordingly, cytology specimens are available for scoring the PD-L1 expression of TCs in patients with advanced LCs, but the PD-L1 staining of ICs and the tumor microenvironment need to be further defined.

We have also found PD-L1 expression (TPS $\geq 1 \%$ ) in $44.4 \%$ of samples $(n=4)$ with EGFR mutations, $63.6 \%$ of samples $(n=7)$ with ALK rearrangement, and $50.0 \%$ of samples $(n=1)$ with ROS1 rearrangement; there was no correlation between the PD-L1 expression scores when compared as ALK, ROS1 rearrangement, and EGFR mutation scores (Table 5). In the KEYNOTE-024 trial for PD-L1 using the 22C3 pharmDx assay, the frequency of overlap between driver oncogene abnormalities (i.e., in ALK or EGFR) and a PD-L1 TPS of at least $50 \%$ was only $6 \%$ (30 of 500). ${ }^{13}$ Lee et al. have found that expression of PD-L1 (TPS $\geq 1 \%$ ) was related to wild-type EGFR, and ROS1 rearrangement was significantly related to high expression of PD-L1. ${ }^{34}$ Conversely, in a previously published study, there was no high expression of PD-L1 in lung adenocarcinoma with EGFR, ALK, or ROS1 mutations, and therapeutic grouping as 
EGFR/ALK/ROS1-mutated, high PD-L1-expression, and biomarker-negative groups was suggested. ${ }^{28}$ Another large metaanalysis revealed that EGFR WT status was significantly related to PD-L1 expression but without ALK or KRAS mutations. ${ }^{35}$ Further studies are needed to establish the real relationship between PD-L1 expression and driver mutations.

The limitations of the current study are related to its retrospective design. Molecular analysis of cytologic samples can be compared with the results of surgical resection samples, and surgical material is better for testing. However, in practice, cytologic samples may limit such a comparison, especially because they represent single samples from patients with advanced disease. The results of our study were consistent with the data in the literature. The concordance of our findings with the results of other institutions will depend on the sample handling methods used and the pathology facilities available.

In our opinion, the evaluation of EGFR, ALK, ROS1, and PD-L1 expression in cytologic materials is reliable, with reasonably good correlation and concordance with histologic specimens, and should be considered for patients without biopsy or surgical material.

Ethics Committee Approval: Ethics committee approval was received for this study from the Ethics Committee of Ege University (Approval No.: 21-2T/55).

Patient Consent for Publication: Written informed consent was obtained from the patients.

Data-sharing Statement: The data that support the findings of this study are available from the corresponding author upon reasonable request.

Author Contributions: Concept - Z.E., D.N.; Design - Z.E., D.N.; Supervision - Z.E., D.N., P.S., A.V.; Resources - Z.E., D.N., A.V.; Materials - Z.E., D.N., P.S., A.V.; Data Collection and/or Processing - Z.E., D.N., P.S.; Analysis and/or Interpretation - Z.E., D.N., P.S.; Literature Review - Z.E., D.N.; Writing - Z.E., D.N., P.S., A.V.; Critical Review - Z.E., D.N., P.S., A.V.

Conflict of Interest: The authors have no conflicts of interest to declare.

Funding: The authors declared that this study had received no financial support.

\section{REFERENCES}

1. Jemal A, Tiwari RC, Murray T, et al. Cancer statistics, 2004. CA Cancer J Clin. 2004;54(1):8-29. [CrossRef]

2. Lastwika KJ, Wilson W, Li QK, et al. Control of PD-L1 expression by oncogenic activation of the AKT-mTOR pathway in non-small cell lung cancer. Cancer Res. 2016;76(2):227-238. [CrossRef]

3. Miyata K, Morita S, Dejima H, et al. Cytological markers for predicting ALK-positive pulmonary adenocarcinoma. Diagn Cytopathol. 2017;45(11):963-970. [CrossRef]

4. Zito Marino FZ, Rossi G, Brunelli M, et al. Diagnosis of anaplastic lymphoma kinase rearrangement in cytological samples through a fluorescence in situ hybridization based assay: cytological smears versus cell blocks. Cancer Cytopathology. 2017;125(5):303-312. [CrossRef]

5. Toh CK. The changing epidemiology of lung cancer. Methods Mol Biol. 2009;472:397411. [CrossRef]

6. Munari E. Expression of programmed cell death ligand 1 in non - small cell lung cancer : comparison between cytologic smears, core biopsies, and whole sections using the SP263 assay. Cancer Cytopathol. 2019;127(1):52-61. [CrossRef]

7. Witkiewicz AK, Dasgupta A, Sammons S, et al. Loss of stromal caveolin-1 expression predicts poor clinical outcome in triple negative and basal-like breast cancers. Cancer Biol Ther. 2010;10(2):135-143. [CrossRef]

8. Tian SK, Killian JK, Rekhtman N, et al. Optimizing workflows and processing of cytologic samples for comprehensive analysis by next-generation sequencing: Memorial
Sloan Kettering Cancer Center experience. Arch Pathol Lab Med. 2016;140(11):12001205. [CrossRef]

9. Rekhtman N, Brandt SM, Sigel CS, et al. Suitability of thoracic cytology for new therapeutic paradigms in non-small cell lung carcinoma: high accuracy of tumor subtyping and feasibility of EGFR and KRAS molecular testing. $J$ Thorac Oncol. 2011;6(3):451-458. [CrossRef]

10. Lindeman NI, Cagle PT, Beasley MB, et al. Molecular testing guideline for selection of lung cancer patients for EGFR and ALK tyrosine kinase inhibitors: guideline from the College of American Pathologists, International Association for the Study of Lung Cancer, and Association for Molecular Pathology. Arch Pathol Lab Med. 2013;137(6):828-860. [CrossRef]

11. Leighl NB, Rekhtman N, Biermann WA, et al. Molecular testing for selection of patients with lung cancer for epidermal growth factor receptor and anaplastic lymphoma kinase tyrosine kinase inhibitors: American Society of Clinical Oncology endorsement of the College of American Pathologists/International Association for the Study of Lung Cancer/Association for Molecular Pathology Guideline. Internat J Clin Oncol. 2014;32(32):3673-3679. [CrossRef]

12. Sadullahoğlu C, Yaman B, Veral A, Nart D. The diagnostic value of cell blocks in liquid-based cytology of bronchial aspiration and bronchial brushing materials. Cytopathology. 2018;29(2):156-162. [CrossRef]

13. Reck M, Rodríguez-Abreu D, Robinson AG, et al. Pembrolizumab versus chemotherapy for PD-L1-positive non-small-cell lung cancer. $N$ Engl $J$ Med. 2016;375(19):1823-1833. [CrossRef]

14. Wang H, Agulnik J, Kasymjanova G, et al. Cytology cell blocks are suitable for immunohistochemical testing for PD-L1 in lung cancer. Ann Oncol. 2018;29(6):14171422. [CrossRef]

15. Stoy SP, Rosen L, Mueller J, Murgu S. Programmed death-ligand 1 testing of lung cancer cytology specimens obtained with bronchoscopy. Cancer Cytopathol. 2018;126(2):122-128. [CrossRef]

16. Pang B, Matthias D, Ong CW, et al. The positive impact of cytological specimens for EGFR mutation testing in non-small cell lung cancer: a single South East Asian laboratory's analysis of 670 cases. Cytopathology. 2012;23(4):229-236. [CrossRef]

17. Bravaccini S, Tumedei MM, Ulivi P, et al. ALK translocation detection in non-small cell lung cancer cytological samples obtained by TBNA or EBUS-TBNA. Cytopathology. 2016;27(2):103-107. [CrossRef]

18. Minca EC, Lanigan CP, Reynolds JP, et al. ALK status testing in non-small-cell lung carcinoma by FISH on ThinPrep slides with cytology material. J Thorac Oncol. 2014;9(4):464-468. [CrossRef]

19. Savic S, Bubendorf L. Common fluorescence in situ hybridization applications in cytology. Arch Pathol Lab Med. 2016;140(12):1323-1330. [CrossRef]

20. Bozzetti C, Nizzoli R, Tiseo M, et al. ALK and ROS1 rearrangements tested by fluorescence in situ hybridization in cytological smears from advanced non-small cell lung cancer patients. Diagn Cytopathol. 2015;43(11):941-946. [CrossRef]

21. Noll B, Wang WL, Gong Y, et al. Programmed death ligand 1 testing in Non - small cell lung carcinoma cytology cell block and aspirate smear preparations. Cancer Cytopathol. 2018;126(5):342-352. [CrossRef]

22. Sakakibara R, Inamura K, Tambo $\mathrm{Y}$, et al. EBUS-TBNA as a promising method for the evaluation of tumor PD-L1 expression in lung cancer. Clin Lung Cancer. 2017;18(5):527-534.e1. [CrossRef]

23. Heymann JJ, Bulman WA, Swinarski D, Pagan CA. PD-L1 expression in non-small cell lung carcinoma: comparison among cytology, small biopsy, and surgical resection specimens. Cancer Cytopathol. 2017;125(12):896-907. [CrossRef]

24. Betz BL, Dixon CA, Weigelin HC, Knoepp SM, Roh MH. The use of stained cytologic direct smears for ALK gene rearrangement analysis of lung adenocarcinoma. Cancer Cytopathol. 2013;121(9):489-499. [CrossRef]

25. Aisner DL, Sams SB. The role of cytology specimens in molecular testing of solid tumors: techniques, limitations, and opportunities. Diagn Cytopathol. 2012;40(6):511524. [CrossRef]

26. Bellevicine C, Malapelle U, de Luca C, Iaccarino A, Troncone G. EGFR analysis: current evidence and future directions. Diagn Cytopathol 2014;42(11):984-992. [CrossRef]

27. Lin CY, Yang CK, Ou YC, et al. Long-term outcome of robotic partial nephrectomy for renal angiomyolipoma. Asian J Surg. 2018;41(2);1-5. [CrossRef]

28. Rangachari D, VanderLaan PA, Shea $\mathrm{M}$, et al. Correlation between classic driver oncogene mutations in EGFR, ALK, or ROS1 and 22C3-PD-L1 $\geq 50 \%$ expression in lung adenocarcinoma. $J$ Thorac Oncol. 2017;12(5):878-883. [CrossRef] 
29. Carter J, Miller JA, Feller-Kopman D, et al. Molecular profiling of malignant pleural effusion in metastatic non-small cell lung carcinoma: the effect of preanalytical factors. Ann Am Thorac Soc. 2017 4(7):1169-1176. (PubMed:[CrossRef]) [CrossRef]

30. McLaughlin J, Han G, Schalper KA, et al. Quantitative assessment of the heterogeneity of PD-L1 expression in non-small-cell lung cancer. JAMA Oncol. 2016;2(1):46-54. [CrossRef]

31. Skov BG, Skov T. Paired comparison of PD-L1 expression on cytologic and histologic specimens From malignancies in the lung assessed with PD-L1 IHC 28-8pharmDx and PD-L1 IHC 22C3pharmDx. Appl Immunohistochem Mol Morphol. 2017;25(7):453-459. [CrossRef]
32. Rehman JA, Han G, Carvajal-Hausdorf DE, et al. Quantitative and pathologist-read comparison of the heterogeneity of programmed death-ligand 1 (PD-L1) expression in non-small cell lung cancer. Mod Pathol. 2017;30(3):340-349. [CrossRef]

33. Rimm DL, Han G, Taube JM, et al. A prospective, multi-institutional, pathologistbased assessment of 4 immunohistochemistry assays for PD-L1 expression in nonsmall cell lung cancer. JAMA Oncol. 2017;3(8):1051-1058. [CrossRef]

34. Russell-goldman E, Kravets S, Dahlberg SE, Sholl LM, Vivero M. Cytologic-histologic correlation of programmed death-ligand 1 immunohistochemistry in lung carcinomas. Cancer Cytopathol. 2018;126(4):253-263. [CrossRef]

35. Pinto D, Schmitt F. Current applications of molecular testing on body cavity fluids. Diagn Cytopathol. 2020;48(9):840-851. [CrossRef] 